\title{
Electromagnon excitations in modulated multiferroics
}

\author{
A. Cano, ${ }^{1, *}$ and E. I. Kats ${ }^{1,2, \text { 田 }}$ \\ ${ }^{1}$ Institut Laue-Langevin, 6 rue Jules Horowitz, B.P. 156, 38042 Grenoble, France \\ ${ }^{2}$ L. D. Landau Institute for Theoretical Physics, RAS, 117940 GSP-1, Moscow, Russia.
}

(Dated: September 14, 2021)

\begin{abstract}
The phenomenological theory of ferroelectricity in spiral magnets presented in [M. Mostovoy, Phys. Rev. Lett. 96, 067601 (2006)] is generalized to describe consistently states with both uniform and modulated-in-space ferroelectric polarizations. A key point in this description is the symmetric part of the magnetoelectric coupling since, although being irrelevant for the uniform component, it plays an essential role for the non-uniform part of the polarization. We illustrate this importance in generic examples of modulated magnetic systems: longitudinal and transverse spin-density wave states and planar cycloidal phase. We show that even in the cases with no uniform ferroelectricity induced, polarization correlation functions follow to the soft magnetic behavior of the system due to the magnetoelectric effect. Our results can be easily generalized for more complicated types of magnetic ordering, and the applications may concern various natural and artificial systems in condensed matter physics (e.g., magnon properties could be extracted from dynamic dielectric response measurements).

PACS numbers: 75.30.Ds, 75.50.-y, 75.25.+z, 62.12.Br
\end{abstract}

The existence of magnetic materials that are also ferroelectrics [1, 2, 3] upsurge recurrent interest to study and to rationalize theoretically various models manifesting this possibility (see e.g. [4, 5, 6, 7]). The recent discovery of ferroelectricity in a family of the so-called frustrated magnets, see e.g. [8], is particularly instructive. In this case, ferroelectricity is a collateral effect of the magnetic ordering that, in contrast to earlier multiferroics, involves modulated structures [9, 10]. The current interest in this new class of ferroelectromagnets is twofold. On one hand the fundamental physics that result from the interplay between the electric and magnetic properties in these systems is not exhausted [10] and, on the other hand, many potential applications arise from this interplay [11].

The appearance of a modulated magnetic structure is accompanied by a distribution of polarization due to the inhomogeneous magnetoelectric effect [12]. This universal effect is similar to so-called flexoelectric effect in liquid crystals [13]. Microscopic studies have focused on the Dzyaloshinskii-Moriya interaction as the main source of the inhomogeneous magnetoelectric effect [4], and this idea seems also to be behind the phenomenological Landau-like approaches carried out until now in relation with ferroelectricity of magnetic origin [5, 14, 15]. As a result, the main attention has been put to only antisymmetric parts of the effect (where the components of the magnetization enter in an antisymmetric combination). However, in a general case, the inhomogeneous magnetoelectric effect has also a non-vanishing symmetric part (see below). This is indeed the natural possibility from the phenomenological point of view [12, 13], whatever the microscopic origin of this symmetric coupling.

In this paper we show that this symmetric part of the inhomogeneous magnetoelectric effect is a key ingredi- ent in the full description of materials exhibiting modulated magnetic structures. When it comes to the uniform polarization, indeed, only the antisymmetric part of the magnetoelectric effect plays a role in the agreement with previous theoretical publications (see e.g., [4, 5, 15], and more details below). However, to describe nonuniform, i.e. modulated in space, polarizations the magnetoelectric effect has to be considered in its full extent. This is precisely the case when addressing the so-called electromagnons, i.e., the normal modes characteristic of ferroelectromagnets that involve both polar modes and magnons in a hybridized way [2, 16].

Electromagnons were theoretically predicted long time ago considering uniformly ordered materials [16]. This consideration has been recently extended to the case of an antiferromagnet that becomes ferroelectric and then, through the inhomogeneous magnetoelectric effect, this induces an incommensurate magnetic structure [17]. The first experimental evidences about electromagnons have been reported for materials in which, showing no instability towards ferroelectricity in the absence of magnetism, it is the appearance of a modulated magnetic structure what make them ferroelectrics [18, 19]. Unfortunately, all theoretical studies performed so far for this latter case have been restricted to only antisymmetric parts of the magnetoelectric coupling [14, 20]. We show below that, contrary to what is implicitly assumed in these works, both symmetric and antisymmetric parts of the inhomogeneous magnetoelectric effect contribute nontrivially to the interplay between the fluctuations of magnetization and polarization in multiferroics.

First of all, let us reconsider the uniform polarization that may arise due to the inhomogeneous magnetoelectric effect. To simplify our presentation and ease the algebra we neglect crystalline field anisotropy (a more realistic 
treatment does not affect our qualitative results). In this case, the magnetoelectric coupling term reads as

$$
F_{E M}=-f_{1} \mathbf{P} \cdot \mathbf{M}(\nabla \cdot \mathbf{M})-f_{2} \mathbf{P} \cdot[\mathbf{M} \times(\nabla \times \mathbf{M})]
$$

where $\mathbf{P}$ is the polarization and $\mathbf{M}$ is the magnetization, and $f_{1}$ and $f_{2}$ are, in general, two different constants. The general form of this term is $-f_{k l, i j} P_{k} M_{i} \partial_{l} M_{j}$ [12], where the magnetoelectric tensor reduces to $f_{k l, i j}=$ $f_{1} \delta_{k i} \delta_{l j}+f_{2}\left(\delta_{k l} \delta_{i j}-\delta_{k j} \delta_{l i}\right)$ in the isotropic case. Let us split the polarization into uniform $\overline{\mathbf{P}}$ and non-uniform (modulated) $\mathbf{P}^{\prime}$ contributions: $\mathbf{P}=\overline{\mathbf{P}}+\mathbf{P}^{\prime}$. From the magnetoelectric term, we then can extract the following contribution to the free energy of the system [21]:

$-f_{k l, i j} \bar{P}_{k} \int d \mathbf{r} M_{i} \partial_{l} M_{j}=\frac{\bar{P}_{k}}{2} \int d \mathbf{r}\left(f_{k l, j i}-f_{k l, i j}\right) M_{i} \partial_{l} M_{j}$

Thus, we see, the uniform polarization indeed couples to the magnetization only through the antisymmetric part of the magnetoelectric tensor. In the isotropic case that is $f_{k l, i j}-f_{k l, j i}=\left(f_{1}+f_{2}\right)\left(\delta_{k i} \delta_{l j}-\delta_{k j} \delta_{l i}\right)$. So, as regards the uniform polarization, the relevant part of the magnetoelectric term (1) can be written as $-\frac{1}{2}\left(f_{1}+f_{2}\right) \overline{\mathbf{P}} \cdot[\mathbf{M}(\nabla \cdot \mathbf{M})-(\mathbf{M} \cdot \nabla) \mathbf{M}]$, which coincides with the expression given in [5]. However, it is important to keep in mind that there are actually two independent contributions, $f_{1}$ and $f_{2}$, to the magnetoelectric coupling term.

Let us now turn our attention to the magnetic features of the modulated magnets discussed in e.g. [10]. As we have mentioned, in these systems ferroelectricity is induced by magnetism and does not appears spontaneously by itself. In addition, the magnetoelectric effect is typically weak in comparison with pure magnetic contributions into the free energy. Therefore, in the first approximation, when determining the magnetic structure and dynamics it is possible to consider the magnetization separately.

Most of these systems first undergo a transition from the magnetically disordered phase to a modulated structure similar to a longitudinal spin-density-wave (LSDW):

$$
\mathbf{M}=\left(M_{1} \cos Q x, 0,0\right),
$$

and then to structure similar to a planar cycloid (PC):

$$
\mathbf{M}=\left(M_{1} \cos Q x, M_{2} \sin Q x, 0\right) .
$$

The dependence on $\mathbf{P}$ of Landau free energy density can be taken as $\frac{1}{2} \chi^{-1} P^{2}+F_{E M}$, where $\chi$ is the dielectric susceptibility in the absence of magnetism [22] and $F_{E M}$ accounts for the magnetoelectric coupling. It can be easily seen that the inhomogeneous magnetoelectric effect (11) implies the appearance of ferroelectricity as a result of the PC structure, but not of the LSDW [5].
A simple way to reproduce this sequence of transitions is by considering Landau free energy density in the form

$$
F_{M}=\frac{a}{2} M^{2}+\frac{b}{4} M^{4}+\frac{1}{2} \sum_{i} M_{i} \hat{L}_{i} M_{i},
$$

where the differential operators $\hat{L}_{i}$ describe the anisotropic softening that gives rise to the modulated structures. For small wavevectors one can approximate $\hat{L}_{i}=c \nabla^{2}$. The free energy (4) then is reduced to a familiar form (see e.g. [23]). Near the wavevector $\mathbf{Q}=(Q, 0,0)$ of the modulated structures $a+\hat{L}_{i} \simeq$ $\alpha_{i}+c_{x} \nabla_{x}^{2}+c \nabla_{\perp}^{2}$. Eq. (4) is then a natural generalization of the free energy considered in [5, 15] that provides the unified description of the spectrum at the relevant wavevectors (i.e., $\mathbf{q}=0$ and $\pm \mathbf{Q}$ ). If $a, b, c>0$, the system shows no instability with respect to uniform magnetizations. Furthermore, if $\alpha_{x}<\alpha_{y}<\alpha_{z}$, in the spirit of arguments presented in [5, 15], one can see that the free energy is minimized by the LSDW modulation in the parameter range $\alpha_{x}<0, \alpha_{y}>0$, while the PC configuration occurs when $\alpha_{x}<0$ and $3 \alpha_{x} \lesssim \alpha_{y} \lesssim \alpha_{x} / 3$ (with $\alpha_{z}>0$ in both cases).

At this point, it is worth making the following comment on the label "improper ferroelectrics" that is frequently put to the systems of our interest (see e.g. [10]). Within above scenario $M_{y}$ or, more precisely, its $\pm \mathbf{Q}$ Fourier components, can be seen as the order parameter of the LSDW-paraelectric $\leftrightarrow$ PC-ferroelectric transition. In the LSDW-paraelectric state Eq. (11) has a term coupling $M_{y}$ and $P_{y}$ linearly so, in view of this linearity, the system should be labeled as proper rather than improper ferroelectric 24]. This is in fact manifested in the corresponding anomalies [5].

As regards the dynamics, we are interested in the lowfrequency excitations of the system. In the magnetically ordered phases, not too close to the transition points, these excitations are associated with variations of the magnetization in which its modulus is conserved. Thus the excitations are described by the Landau-Lifshitz equation [23]:

$$
\dot{\mathbf{M}}=\gamma \mathbf{M} \times \mathbf{H}_{\mathrm{eff}},
$$

where $\gamma$ is a constant and $\mathbf{H}_{\text {eff }}=\left(\hat{L}_{x} M_{x}, \hat{L}_{y} M_{y}, \hat{L}_{z} M_{z}\right)$.

The dispersion relations and correlation functions of our interest are obtained by linearizing this equation about the corresponding configurations of equilibrium. Then it is convenient to consider first of all the LSDW structure (2), and then a virtual transverse spin-densitywave (TSDW) modulation:

$$
\mathbf{M}=\left(0, M_{2} \sin Q x, 0\right) .
$$

This would be the structure obtained from (4) if, contrary to what we assumed for the LSDW, $\alpha_{y}$ becomes negative with $\alpha_{x}>0$. This structure TSDW is of interest in its own right and besides, it turns out that the 
low-lying normal modes of the PC case can be found as the superposition of the normal modes associated with LSDW and TSDW structures. This notably simplifies the corresponding calculations.

Skipping a large amount of tedious (although straightforward and simple) algebra, we come to the following results. They are obtained under the same assumptions as those for the static problem. That is, assuming that the anharmonicity is weak and that the spatial dispersion such that the generalized stiffness presents well defined minima only at the wavevectors $\mathbf{q}=0, \pm \mathbf{Q}$. This allows us to decouple the modes $\mathbf{q}$ and $\mathbf{q} \pm \mathbf{Q}$ from the rest [25]. To our purposes, higher-order satellites can be neglected.

As it could be expected, the low-lying modes of the LSDW represent independent oscillations of the magnetization along the $y$ and $z$ axes. The corresponding dispersion relations are such that

$$
\omega_{y, z}^{2}(\mathbf{q})= \begin{cases}2 m_{x}^{2}\left(\alpha_{z, y}-\alpha_{x}+c_{x} q_{x}^{2}+c q_{\perp}^{2}\right)\left(-\alpha_{x}+c q^{2}\right), & q \rightarrow 0, \\ m_{x}^{2}\left(\widetilde{\alpha}_{z, y}-2 \alpha_{x}+c q^{\prime 2}\right)\left(\alpha_{y, z}-\alpha_{x}+c_{x} q_{x}^{\prime 2}+c q_{\perp}^{\prime 2}\right), & \mathbf{q}=\mathbf{Q}+\mathbf{q}^{\prime}\left(q^{\prime} \rightarrow 0\right)\end{cases}
$$

Here $m_{x}=\gamma M_{1} / 2$ and the quantities $\widetilde{\alpha}_{i}$ are defined such that $\hat{L}_{i} e^{ \pm i 2 Q x}=\widetilde{\alpha}_{i} e^{ \pm i 2 Q x}$. It is worth noticing that the closer the transition is, the smaller is the gap obtained at small wavevectors. Not only because of the smallness of $m_{x}$, what influences trivially on the whole spectrum, but also because $\alpha_{x} \rightarrow 0$. In fact, very close to the transition point $\omega_{y, z}(q \rightarrow 0) \sim q$. The gap obtained close to the wavevector of the modulation, on the contrary, is not so sensitive to the smallness of $\alpha_{x}$.

Similar expressions are found for the TSDW structure (6). In this case, the low-lying modes are associated with oscillations of the magnetization along the $x$ and $z$ axes. Their dispersion relations can be obtained from (7) by replacing $y \leftrightarrow x$ in $m_{x}, \alpha_{i}$ and $\widetilde{\alpha}_{i}$.

As we have mentioned, these results for the LSDW and TSDW structures merge in the PC case. Three lowlying normal modes are found such that the modulus of the magnetization is conserved in this latter structure. The modes associated with oscillations along the $x$ and $y$ axes coincide with that obtained previously for the TSDW and LSDW modulations respectively. Consequently, they have the same dispersion relations. The mode representing oscillations along the $z$ axis, however, is composed by the modes associated with the same type of oscillation in the TSDW and LSDW cases. In consequence, its dispersion relation can be expressed such that $\omega_{z, \mathrm{PC}}^{2}(\mathbf{q})=\omega_{z, \mathrm{LSDW}}^{2}(\mathbf{q})+\omega_{z, \mathrm{TSDW}}^{2}(\mathbf{q})$.

Let us now turn our attention to the fluctuations. Since the softness of the system is in its magnetic part, fluctuations of the magnetization $\delta \mathbf{M}$ will play the main role. Once Eq. (5) is linearized, the corresponding correlation functions:

$$
M_{i j}(\mathbf{q}) \equiv\left\langle\delta M_{i}(\mathbf{q}, \omega) \delta M_{j}(-\mathbf{q},-\omega)\right\rangle,
$$

can be found with the aid of the fluctuation-dissipation theorem 23]. Within a first approximation, the polarization will follow these fluctuations in accordance with the magnetoelectric coupling (1). This results in fluc- tuations of the polarization $\delta \mathbf{P}$ of magnetic origin, i.e., electromagnons, whose correlation functions:

$$
P_{i j}(\mathbf{q}) \equiv\left\langle\delta P_{i}(\mathbf{q}, \omega) \delta P_{j}(-\mathbf{q},-\omega)\right\rangle
$$

can be expressed in terms of the previous quantities (8). The hybridization between magnons and polar modes reflects also in the form of cross correlation functions $\left\langle\delta P_{i} \delta M_{j}\right\rangle$. Within our approximation, these cross correlations can also be expressed in terms of the quantities (8). But since they essentially reveal the same information (see e.g. 26]), we omit them in the following.

As regards magnetic correlation functions, the "nondiagonal" components are zero for the structures of our interest $\left(M_{i j}=0\right.$ if $\left.i \neq j\right)$. In addition, $M_{x x}=0$ in the LSDW case while $M_{y y}=0$ in the TSDW one. Close to the relevant wavevectors, the non-vanishing correlation functions are such that $M_{i i}^{-1}(\mathbf{q}) \propto \tau\left\{\left[\omega^{2}-\omega_{i}^{2}(\mathbf{q})\right]^{2}+\right.$ $\left.\tau^{-2} \omega^{2}\right\}$, where $\tau$ is the (phenomenological) relaxation time of the corresponding oscillations.

The fluctuations of the polarization of magnetic origin can be described in terms of the quantities $M_{i i}$. These fluctuations are such that the soft magnetic behavior at $\mathbf{q} \approx \pm \mathbf{Q}$ reflects in dipole polarization excitations with small wavevectors. And reversely, the soft magnetic response at $q \rightarrow 0$ reflects in polarization excitations with $\mathbf{q} \approx \pm \mathbf{Q}$, as can be seen in further formulas. Note also that the magnetoelectric coefficients $f_{1}$ and $f_{2}$ enter separately in the following results. That is, not only through the combination $f_{1}+f_{2}$ that determines the antisymmetric part of the magnetoelectric effect and, consequently, the space average of the polarization of the system. (The q-dependence associated with $\chi[22]$ is implicit in these formulas.) 
In the LSDW case we have

$$
\begin{aligned}
& P_{x x}(\mathbf{q}) \sim f_{1}^{2}\left\{q_{y}^{2}\left[M_{y y}(\mathbf{q}-\mathbf{Q})+M_{y y}(\mathbf{q}+\mathbf{Q})\right]\right. \\
&\left.\quad+q_{z}^{2}\left[M_{z z}(\mathbf{q}-\mathbf{Q})+M_{z z}(\mathbf{q}+\mathbf{Q})\right]\right\}, \\
& P_{y y}(\mathbf{q}) \sim g_{-2}^{2}(\mathbf{q}) M_{y y}(\mathbf{q}-\mathbf{Q})+g_{+2}^{2}(\mathbf{q}) M_{y y}(\mathbf{q}+\mathbf{Q}), \\
& P_{z z}(\mathbf{q}) \sim g_{-2}^{2}(\mathbf{q}) M_{z z}(\mathbf{q}-\mathbf{Q})+g_{+2}^{2}(\mathbf{q}) M_{z z}(\mathbf{q}+\mathbf{Q}), \\
& P_{x y}(\mathbf{q}) \sim f_{1} q_{y}\left[g_{-2}(\mathbf{q}) M_{y y}(\mathbf{q}-\mathbf{Q})-g_{+2}(\mathbf{q}) M_{y y}(\mathbf{q}+\mathbf{Q})\right], \\
& P_{x z}(\mathbf{q}) \sim f_{1} q_{y}\left[g_{-2}(\mathbf{q}) M_{z z}(\mathbf{q}-\mathbf{Q})-g_{+2}(\mathbf{q}) M_{z z}(\mathbf{q}+\mathbf{Q})\right], \\
& P_{y z}(\mathbf{q})=0 .
\end{aligned}
$$

where $g_{ \pm 2}(\mathbf{q})=\left(f_{1}+f_{2}\right) Q \pm f_{2} q_{x}$. In the case of a pure symmetric coupling $\left(f_{1}=-f_{2}\right)$, with no DzyaloshinksiiMoriya-like contributions, all these correlation functions turn out to be $\propto q^{2}$. In consequence, the eventual importance of fluctuations of the polarization with small wavevectors is conditioned to the existence of a nonvanishing antisymmetric part in the magnetoelectric coupling. In any case, fluctuations with wavevectors $\mathbf{q} \approx \pm \mathbf{Q}$ are important irrespective to the above vanishing (they are entirely due to $f_{1} \neq 0$ ). These "magnetic" softenings of optical phonons in the LSDW paraelectric state can be used to measure the corresponding coefficients $f_{1}$ and $f_{2}$. This soft behavior in the absence of ferroelectricity has in fact been observed in 19 .

Similarly, for the TSDW structure we find that

$$
\begin{aligned}
P_{x x}(\mathbf{q}) \sim & f_{2}^{2} q_{y}^{2}\left[M_{x x}(\mathbf{q}-\mathbf{Q})+M_{x x}(\mathbf{q}+\mathbf{Q})\right] \\
P_{y y}(\mathbf{q}) \sim & g_{-1}^{2}(\mathbf{q}) M_{x x}(\mathbf{q}-\mathbf{Q})+g_{+1}^{2}(\mathbf{q}) M_{x x}(\mathbf{q}+\mathbf{Q}) \\
& +f_{1}^{2} q_{z}^{2}\left[M_{z z}(\mathbf{q}-\mathbf{Q})+M_{z z}(\mathbf{q}+\mathbf{Q})\right] \\
P_{z z}(\mathbf{q}) \sim & f_{2}^{2} q_{y}^{2}\left[M_{z z}(\mathbf{q}-\mathbf{Q})+M_{z z}(\mathbf{q}+\mathbf{Q})\right] \\
P_{x y}(\mathbf{q}) \sim & f_{2} q_{y}\left[g_{-1}(\mathbf{q}) M_{x x}(\mathbf{q}-\mathbf{Q})-g_{+1}(\mathbf{q}) M_{x x}(\mathbf{q}+\mathbf{Q})\right] \\
P_{x z}(\mathbf{q})= & 0 \\
P_{y z}(\mathbf{q}) \sim & -f_{1} f_{2} q_{y} q_{z}\left[M_{z z}(\mathbf{q}-\mathbf{Q})+M_{z z}(\mathbf{q}+\mathbf{Q})\right]
\end{aligned}
$$

where $g_{ \pm 1}(\mathbf{q})=\left(f_{1}+f_{2}\right) Q \pm f_{1} q_{x}$. As in the previous case, the relevance of fluctuations of the polarization with small wavevectors is made conditional on the antisymmetric part of the magnetoelectric coupling while the relevance of fluctuations with wavevectros close to that of the modulated structure is not.

Finally, armed with the knowledge of correlation functions for LSDW and TSDW structures, we are in the position to calculate the polarization correlation functions in the PC case as $P_{i j}^{\mathrm{LSDW}}+P_{i j}^{\mathrm{TSDW}}+\Delta P_{i j}$. Here the first two terms represent the correlation functions obtained in for the LSDW and TSDW modulations respectively, and the third one is

$$
\begin{aligned}
& \Delta P_{x x}(\mathbf{q}) \sim q_{x}^{2}\left[f_{1}^{2} \Pi_{x}(\mathbf{q}, \mathbf{Q})+f_{2}^{2} \Pi_{y}(\mathbf{q}, \mathbf{Q})\right], \\
& \Delta P_{y y}(\mathbf{q}) \sim q_{y}^{2}\left[f_{2}^{2} \Pi_{x}(\mathbf{q}, \mathbf{Q})+f_{1}^{2} \Pi_{y}(\mathbf{q}, \mathbf{Q})\right], \\
& \Delta P_{z z}(\mathbf{q}) \sim f_{2}^{2} q_{z}^{2}\left[\Pi_{x}(\mathbf{q}, \mathbf{Q})+\Pi_{y}(\mathbf{q}, \mathbf{Q})\right], \\
& \Delta P_{x y}(\mathbf{q}) \sim f_{1} f_{2} q_{x} q_{y}\left[\Pi_{x}(\mathbf{q}, \mathbf{Q})+\Pi_{y}(\mathbf{q}, \mathbf{Q})\right], \\
& \Delta P_{x z}(\mathbf{q}) \sim f_{1} f_{2} q_{x} q_{z}\left[\Pi_{x}(\mathbf{q}, \mathbf{Q})+\Pi_{y}(\mathbf{q}, \mathbf{Q})\right], \\
& \Delta P_{y z}(\mathbf{q}) \sim f_{1} f_{2} q_{y} q_{z}\left[\Pi_{x}(\mathbf{q}, \mathbf{Q})+\Pi_{y}(\mathbf{q}, \mathbf{Q})\right] .
\end{aligned}
$$

where $\Pi_{i}(\mathbf{q}, \mathbf{Q})=m_{i}^{2}\left[M_{i i}(\mathbf{q}-\mathbf{Q})+M_{i i}(\mathbf{q}+\mathbf{Q})\right]$. These additional contributions are relevant for the fluctuations with wavevectors close to $\pm \mathbf{Q}$. It is worth noticing that, within our approximations, there are no contributions $\propto m_{1} m_{2}$ to these correlations functions.

In summary, we have presented the key aspects of the phenomenological theory of ferroelectricity of magnetic origin that allow one to treat consistently, on the same footing, uniform and non-uniform polarizations. One of its key points is the accounting for all the possible contributions, symmetric and antisymmetric ones, to the inhomogeneous magnetoelectric effect. Considering the most representative modulated magnetic structures, we have employed this theory to study the corresponding fluctuations of magnetization and polarization. Our main findings are the following. i) The aforementioned fluctuations turn out to be interdependent as long as magnetic order appears, irrespective of whether this order induces an uniform polarization or not. This implies that ferroelectricity may not be strictly necessary when addressing applications based on such an interplay. ii) The softness of the system reveals in the form of large fluctuations at both, small wavevectors and wavevectors close to that of the modulated structure. At small wavevectors, the fluctuations of the polarization of magnetic origin can be associated with the antisymmetric part of the magnetoelectric effect (that eventually gives rise to the uniform polarization of the system). At wavevectors close to that of the modulated structure, however, the situation is different. In this case, the fluctuations are due to both symmetric and antisymmetric parts of the effect. Consequently, in view of the relativistic nature of the antisymmetric part, one can expect here a leading role of the symmetric contribution (which has its origin in the exchange interaction).

We acknowledge very fruitful discussions with L.P. Regnault, I.E. Dzyaloshinskii and A.P. Levanyuk. A.C. was supported by a postdoctoral fellowship from Fundación Ramón Areces.

* Electronic address: cano@ill.fr

† Electronic address: kats@ill.fr

[1] K. Aizu, Phys. Rev. B 2, 754 (1970). 
[2] G.A. Smolenskii and I.E. Chupis, Usp. Fiz. Nauk 137 415 (1982) [Sov. Phys. Usp. 25, 475 (1982)].

[3] Y.N. Venevtsev, V.V. Gagulin, Ferroelectrics 162, 23 (1994); H. Schmid, Ferroelectrics 162, 317 (1994).

[4] H. Katsura, N. Nagaosa and A.V. Balatsky, Phys. Rev. Lett. 95057205 (2005); I.A. Sergienko and D.E. Dagotto, Phys. Rev. B 73094434 (2006).

[5] M. Mostovoy, Phys. Rev. Lett. 96, 067601 (2006).

[6] D.I. Khomskii, J. Magn. and Magn. Mat. 306, 1 (2006).

[7] J.J. Betouras, G. Giovannetti and J van den Brink, Phys. Rev. Lett. 98, 257602 (2007); A.B. Harris, Phys. Rev. B 76, 054447 (2007).

[8] T. Kimura et al., Phys. Rev. B 68, 060403(R) (2003); T. Kimura et al., Nature (London) 426, 55 (2003); T. Goto et al., Phys. Rev. Lett. 92, 257201 (2004); N. Hur et al., Nature (London) 429, 392 (2004).

[9] I.E. Dzyaloshinskii, Zh. Eksp. Teor. Fiz. 46, 1420 (1964) [Sov. Phys. JETP 19, 960 (1964)]; 47336 (1964) [20, 223 (1965)];

[10] M. Fiebig, J. Phys. D 38, R123 (2005); N.A. Spaldin and M. Fiebig, Science 309, 391 (2005); R. Ramesh and N.A. Spaldin, Nature Mater. 6, 21 (2007); S.W. Cheong and M. Mostovoy, Nature Mater. 6, 13 (2007).

[11] J.F. Scott, Science 315, 954 (2007).

[12] V.G. Bar'yakhtar, V.A. L'vov and D.A. Yablonskii, Pis'ma Zh. Eksp. Teor. Fiz. 37565 (1983) [JETP Lett. 37, 673 (1983)].

[13] P.G. de Gennes and J. Prost, The Physics of Liquid Crystals (Clarendon, Oxford, 1993).

[14] S. Tewari et al., arXiv:0710.4551v1.

[15] C. Zhang et al., arXiv:0710.4550v2.

[16] V.G. Bar'yakhtar and I.E. Chupis, [Sov. Phys. Solid State
11, 2628 (1970)]; G.A. Maugin, Phys. Rev. B 23, 4608 (1981); D.R. Tilley and J.F. Scott, ibid. 25, 3251 (1982).

[17] R. de Sousa and J.E. Moore, Phys. Rev. B 77, 012406 (2008).

[18] A. Pimenov et al., Nature Phys. 297 (2006); A.B. Sushkov et al., Phys. Rev. Lett. 98, 027202 (2007); N. Kida et al., arXiv:0711.2733v1.

[19] D. Senff et al., Phys. Rev. Lett. 98, 137206 (2007); R. Valdes-Aguilar et al., Phys. Rev. B 76, 060404(R) (2007).

[20] H. Katsura, A.V. Balatsky and N. Nagaosa, Phys. Rev. Lett. 980272203 (2007).

[21] To derive the latter expression we transform the magnetoelectric tensor into $\left(f_{k l, i j}+f_{k l, i j}\right) / 2$ and integrate by parts one of the resulting terms.

[22] Strictly speaking, in the absence of magnetism we should write $\frac{1}{2}\left(\chi_{0}^{-1} P^{2}-\mathbf{E} \cdot \mathbf{P}\right)$, where $\chi_{0}$ is a constant and the field $\mathbf{E}$ is caused by inhomogeneous distributions of $\mathbf{P}$. That is, the electric field obtained from the equations of electrostatics $\nabla \times \mathbf{E}=0$ and $\nabla \cdot(\mathbf{E}+4 \pi \mathbf{P})=0$. This results into the susceptibility $\chi$.

[23] L.D. Landau and E.M. Lifshitz, Statistical Physics, Parts 1 and 2 (Pergamon Press, Oxford, 1980).

[24] B.A. Strukov and A.P. Levanyuk, Ferroelectric Phenomena in Crystals (Springer-Verlag, Berlin, 1998).

[25] YuA. Izyumov, Usp. Fiz. Nauk 144, 439 (1984) [Sov. Phys. Usp. 27, 845 (1984)]; A.P. Levanyuk, in Incommensurate Phases in Dielectrics, edited by R. Blinc and A.P. Levanyuk (North-Holland, Amsterdam, 1986).

[26] V.L. Ginzburg, A.P. Levanyuk and A.A. Sobyanin, Phys. Rep. 57, 151 (1980). 